\title{
Directed Transport in classical and quantum chaotic billiards
}

\author{
W Acevedo and T Dittrich \\ Departamento de Física, Universidad Nacional de Colombia, and \\ CeiBA - Complejidad, Bogotá D.C., Colombia \\ E-mail: jwacevedov@unal.edu.co,tdittrich@unal.edu.co
}

\begin{abstract}
We construct an autonomous chaotic Hamiltonian ratchet as a channel billiard subdivided by equidistant walls attached perpendicularly to one side of the channel, leaving an opening on the opposite side. A static homogeneous magnetic field penetrating the billiard breaks time-reversal invariance and renders the classical motion partially chaotic. We show that the classical dynamics exhibits directed transport, owing to the asymmetric distribution of regular regions in phase space. The billiard is quantized by a numerical method based on a finite-element algorithm combined with the Landau gauge and the Bloch formalism for periodic potentials. We discuss features of the billiard eigenstates such as node lines and vortices in the probability flow. Evidence for directed quantum transport, inherited from the corresponding features of the classical dynamics, is presented in terms of level-velocity statistics.
\end{abstract}

PACS numbers: $05.45 . \mathrm{Mt}, 73.23 . \mathrm{Ad}$, 75.47.Jn 


\section{Introduction}

Within two decades, the concept of ratchets has undergone a remarkable process of refinements and transformations. Originating in an attempt to understand the functioning of motor molecules in terms of fundamental principles of statistical mechanics, ratchets have first been conceived as overdamped stochastic systems, modelling molecular motion in the intracellular medium [1, 2, 3, 4]. Inertia terms have subsequently been included in the equations of motion to allow for a more complex dynamics than mere relaxation [5]. As a more radical step, friction has then been neglected altogether, giving way to Hamiltonian ratchets [6, 7]. In replacing viscous forces, another mechanism to break time-reversal invariance (TRI) had to be introduced. It was found in a periodic external driving with asymmetric time dependence, for example with a sawtooth profile similar to that of the static ratchet potential [8]. In this way, the external driving attained three functions that are crucial for the behaviour of Hamiltonian ratchets, namely (i), breaking TRI, (ii), rendering the dynamics chaotic, and (iii), injecting or absorbing energy in case that a finite mean potential gradient be present.

Directed transport in classical as well as quantum Hamiltonian ratchets has been studied in great detail in recent years [6, 7, 8, which led to various basic insights such as a sum rule for transport, as a lemma of the conservation of phase-space volume, dynamical mechanisms that generate directed currents, and the fingerprints of these currents in the band spectra of quantum ratchets.

It is then tempting to ask whether ratchet-like transport can be obtained even abandoning the time-dependent external force, thus making the last step towards autonomous Hamiltonian ratchets. In view of the functions of the driving mentioned above, it is clear that alternative means are then needed to destroy TRI and to produce a chaotic dynamics. This latter requirement implies in particular that a minimum number of two degrees of freedom is necessary. By a fortunate coincidence, both conditions can be fulfilled simultaneously if a static magnetic field is imposed that penetrates the plane of motion perpendicularly (assuming that the particles at hand carry an electric charge).

In this paper we study periodic billiards immersed in a homogeneous magnetic field. In order to remain as close as possible to one-dimensional ratchet models, we construct them as quasi-one-dimensional billiard chains. In fact it is sufficient to consider a straight channel with equidistant walls or diaphragms attached orthogonally to one sidewall of the channel, leaving a sequence of gaps on the opposite side. In fact, this configuration has already been conceived as a model for quantum chaos [9]. Periodic billiards without magnetic field, where classically chaotic motion is induced instead by curved walls, have been studied to elucidate, in particular, fingerprints of chaos in the band structure and energy eigenstates [11].

In presence of the magnetic field, straight walls at right angles are sufficient to render the motion of charged particles chaotic, at least in parts of phase space, in a billiard that would otherwise be integrable [12, 14, 15]. Upon quantization, classically 
chaotic billiards in a magnetic field exhibit signatures of chaos in observable phenomena like the magnetic susceptibility [16]. Particularly close in spirit to our work are studies of magnetotransport in ballistic two-dimensional electron gases moving in antidot superlattices [17]. Features of the classical nonlinear dynamics are reflected in anomalies both of the longitudinal magnetoresistance [18] and of the Hall effect [19] that had previously been observed experimentally [20. A remarkable duality between the interior (quantum dot) and the exterior (antidot) states of electron billiards has been elucidated in [21].

Here, by contrast, we are interested in directed (dc) transport without applied external voltage. It is the broken symmetry in the transverse direction together with the breaking of TRI by the magnetic field that leads to an effective ratchet behavior. A trajectory transporting, say, to the right, is then generally not compensated by a corresponding symmetry-related trajectory transporting to the left. Net directed currents ensue. This effect has been studied on the classical level for a billiard chain very similar to our model, though with a smoothly curved boundary that would induce chaos even in the absence of a magnetic field [22]. Experimentally, ratchet effects have been observed in quasi-one-dimensional semiconductor superlattices, but subject to an external periodic driving [23].

In driven ratchets, even pumping in the sense of directed transport "uphill" is possible, as well as stationary currents "downhill" [7]. In our case, the fact that we are dealing with an autonomous system prevents this option. The lack of an inexhaustible energy source/sink coupled to the system implies that the ratchet effect depends much more sensitively on the absence of a mean potential gradient than in driven systems. To be sure, this is true only for an infinitely extended billiard. For a section of finite length the zero-gradient transport properties may well affect the response to an external voltage and will then be reflected in the system's magnetotransport properties. This aspect is beyond the scope of the present paper, but we briefly comment on it in the conclusion.

In Section 2, we define the geometry of our magnetic billiard and construct the classical dynamics as a discrete map based on a Poincaré surface of section. We demonstrate that directed currents occur under the same conditions and depend on the same mechanisms (regular regions embedded in the chaotic sea that are not symmetric under momentum inversion) as in driven ratchets. The asymptotic behavior for weak vs. strong magnetic field and for wide vs. narrow openings between the billiard compartments is discussed and its implications for transport are pointed out. For a large region in parameter space we can even give an approximate analytic expression for the net current.

Section 3 is dedicated to the quantization of the magnetic billiard. In some limits (e.g., square billiard, empty channel, Landau states in the 2-dim. electron gas), analytical solutions are available. They serve as reference for the calibration of the numerical approaches we have to resort to in general. We briefly explain the quantization algorithm of our choice, a finite element method, and how it has to be complemented with Bloch theory as it applies to the periodic billiard. We discuss features of general 
interest of the billiard's band spectrum, e.g., the traces of Landau levels and diabatic bands associated to invariant manifolds of the classical phase space, as well as of the eigenstates, such as edge states and vortices in the quantum flow. Evidence of directed currents is presented in terms of level-velocity statistics.

We conclude in Section 4 considering possible extensions of this work and open questions related to them, like relations to the quantum Hall effect, the construction of pumps from finite sections of the billiard chain, and the effects of an external driving.

\section{Classical magnetic billiard}

\subsection{Billiard geometry and trajectories}

The billiard is constructed as a straight channel of width $w$ with equidistant barriers of length $a$, separated by a distance $l$ from each other and attached at right angles to one side (figure 11) [24, 25]. It is threaded by a homogeneous magnetic field $B$ perpendicular to the plane of the billiard. We shall only consider the case of particles with unit mass and charge $(m=q=1)$ moving in a square unit cell $(l=w=1)$ so that barrier size $a$ and cyclotron radius $r_{\mathrm{c}}=p / B$, with $p$, the constant modulus of the momentum, remain as control parameters. The direction of the magnetic field is chosen such that for $r_{\mathrm{c}}>0$, clockwise motion on circular-arc-shaped trajectories results. Upon hitting a wall, trajectories undergo specular reflection.
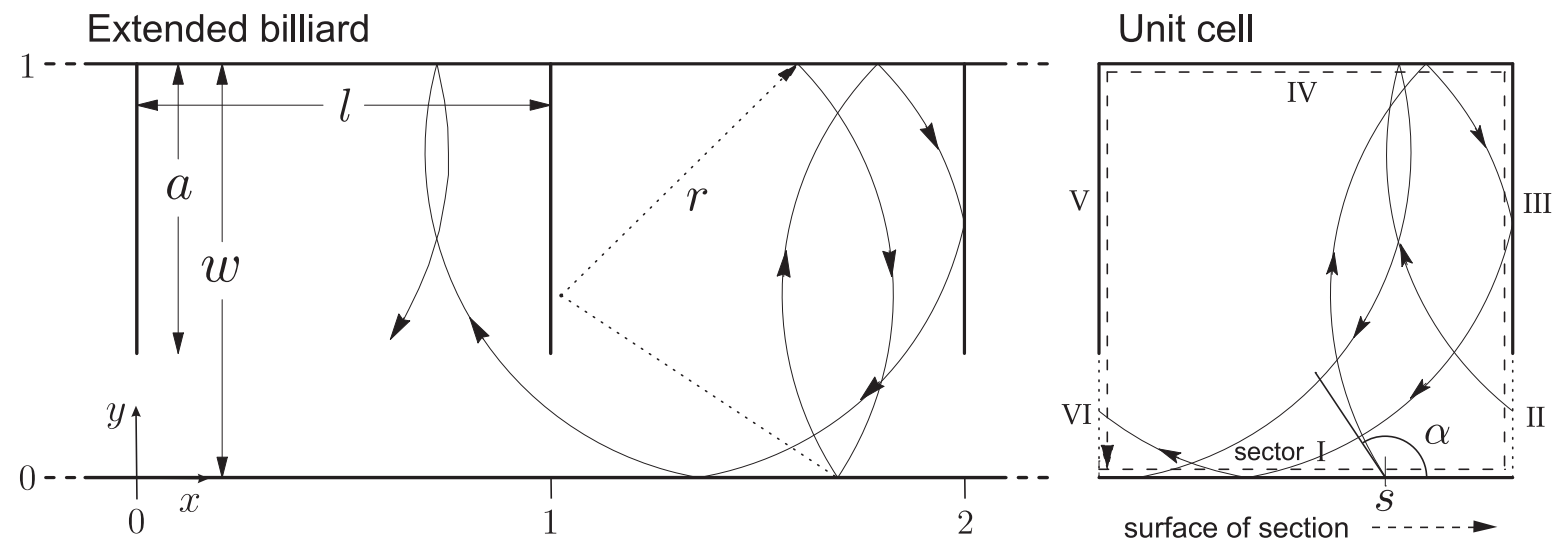

Figure 1. Geometry of the billiard.

\subsection{Symmetries}

The spatio-temporal symmetries of the billiard will attain their full impact only in the context of quantization, but for completeness we present them already here.

Considering only the geometry of the walls, the billiard is periodic in $x$ with period 1,

$$
V(x+1, y)=V(x, y),
$$


and invariant under reflection in $x$,

$$
V(-x, y)=V(x, y) \text {. }
$$

Together with (11) this implies reflection symmetry with respect to every $x_{n}=n / 2, n$ integer.

With a non-zero magnetic field, the Hamiltonian retains the periodicity (1) while parity (2) is broken, as is of course time-reversal invariance. Limiting cases of interest are the empty channel, $a=0$, where the dynamics is invariant under continuous translations in $x$, and the closed square billiard which in the presence of a magnetic field retains a $C_{4}$ rotation symmetry (without reflection) [26].

\subsection{Poincaré map, phase-space structure and limiting cases}

A Poincaré surface of section is constructed on basis of a closed line following the entire circumference of the unit cell but shifted inwards by an infinitesimal amount, see figure 1. It is parameterized by the Birkhoff coordinates $s$ (position along the circumference, measured counterclockwise from the origin) and $p_{s}=\cos \alpha$ (tangential momentum after crossing the surface). The elementary geometry of the trajectories (circular arcs connected by specular reflection at the walls) allows us to formulate the dynamics as a Poincaré map in terms of a relatively simple algorithm, see appendix A.

According to the different types of boundary conditions around the unit cell, we subdivide the Poincaré surface of section into six subsections I to VI (lower wall, right opening, right wall, upper wall, left wall, left opening). Figure 2 shows a typical phase portrait, indicating a mixed dynamics with regular islands immersed in a chaotic sea and continuous sets of marginally stable orbits inherited from the integrable motion that dominates in certain limits, as discussed in the sequel.

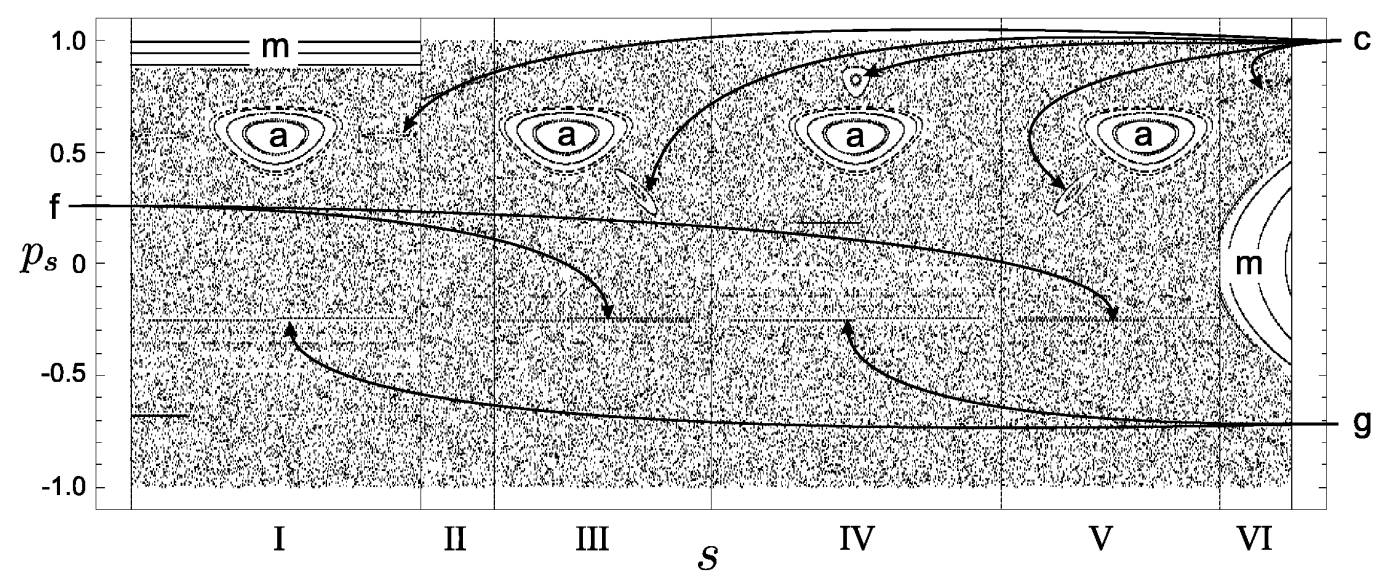

Figure 2. Phase portrait for $r_{\mathrm{c}}=2$ and $a=0.75$. Lettered features correspond to the orbit types shown in figure 3 below.

As the billiard boundary does not include any curved section, it is clear that for vanishing magnetic field (divergent radius of curvature $r_{\mathrm{c}}$ of the trajectories), the 
dynamics cannot be chaotic. The presence of openings between the cells however render the phase-space topology of the unit cell multiply connected and therefore the motion pseudo-integrable in this limit [27, 28, 29]. In this case, in fact, the billiard can be extended to the entire $(x, y)$-plane by repeated reflection at the upper and lower horizontal walls, and thus reduces to an infinite rectangular lattice of straight-wall segments ("barrier billiard" [28]). This feature is lost for finite $r_{\mathrm{c}}$, as the curvature changes sign upon reflection.
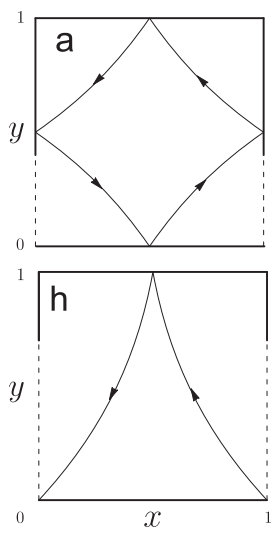
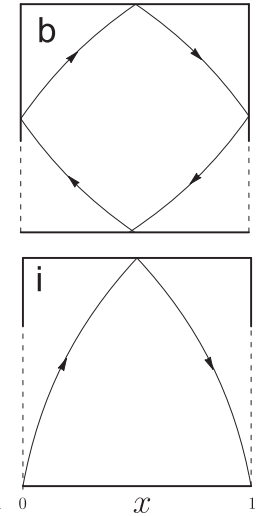
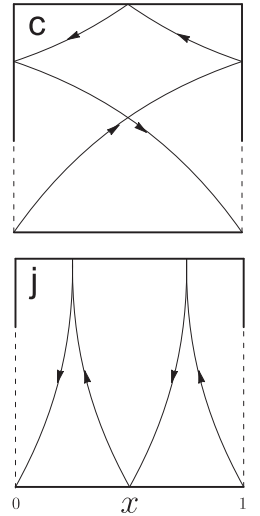
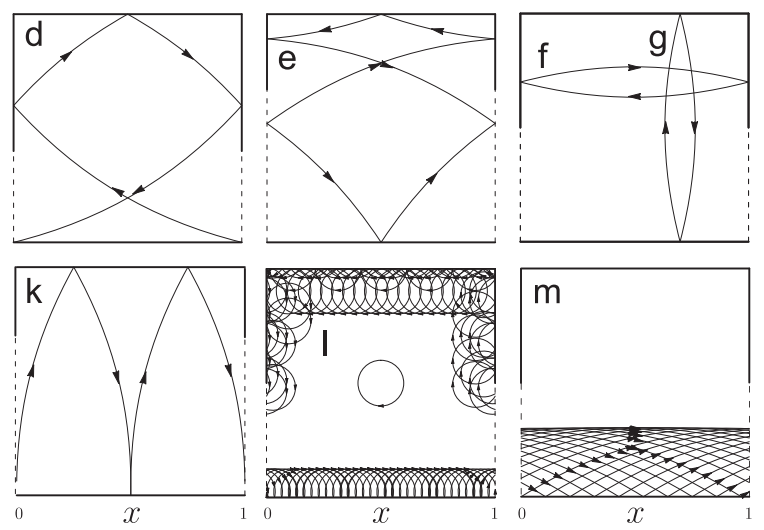

Figure 3. Some periodic orbits for $r_{\mathrm{c}}=2(\mathrm{a}-\mathrm{k})$, orbits for $r_{\mathrm{c}}=0.1$ (l) and noncontractible trajectories $(\mathrm{m})$. The location of some of these orbits is indicated by the same letters in the phase portrait, figure 2 ,

Pseudo-integrability is approached continuously via the Kolmogorov-Arnol'd-Moser scenario [30] for $r_{\mathrm{c}} \rightarrow \infty$, see figure 44d. For $r_{\mathrm{c}} \rightarrow 0$ (the high-field limit), on the other hand, trajectories touching the boundary become skipping orbits [21, 31, 33], while those inside the billiard reduce to full circles (figure 31). In this limit the system thus approaches integrability, but in a highly singular manner [25], cf. figure 4a.
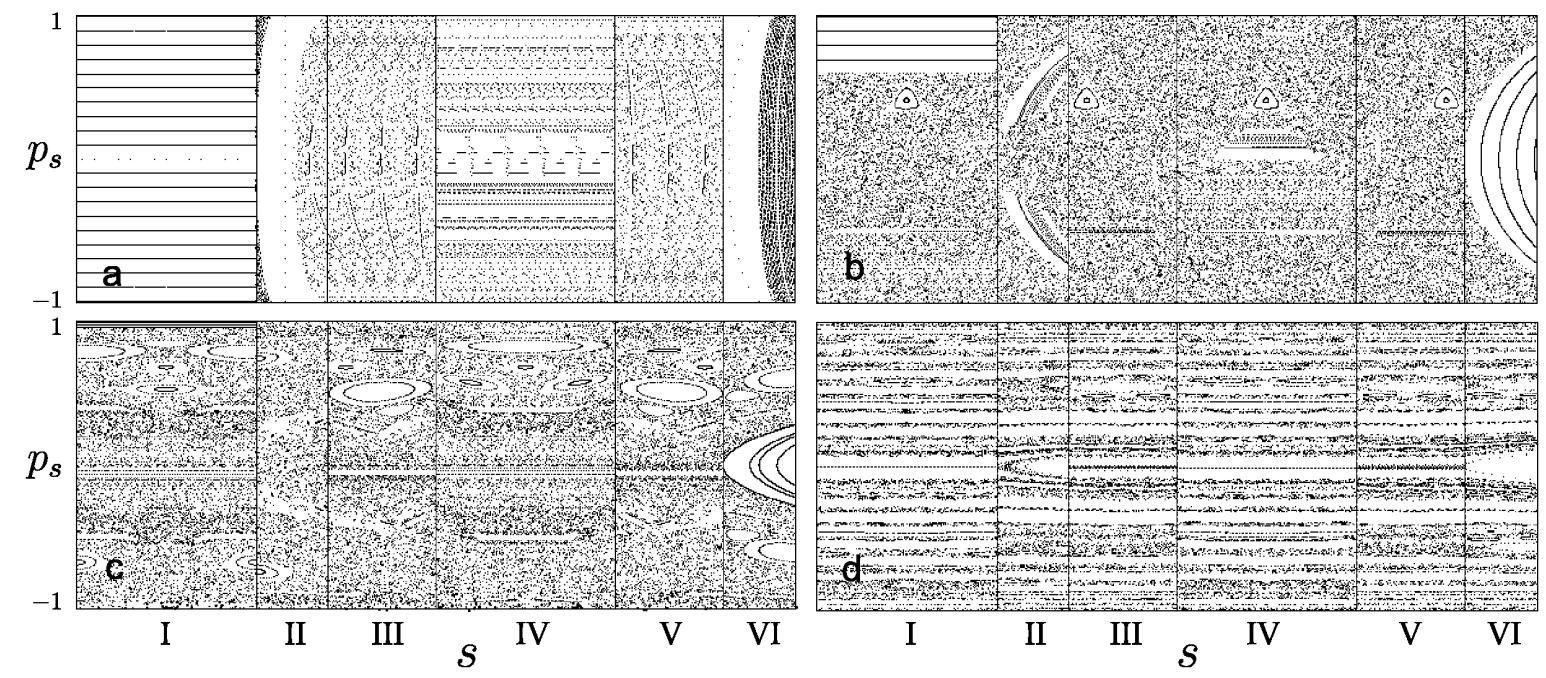

Figure 4. Phase-space portraits for $a=0.6$ and $r_{\mathrm{c}}=0.1$ (a), 1 (b), 10 (c), 100 (d). 
Concerning the wall size $a$, the limit $a=0$, that is the free channel billiard, is integrable irrespective of the cyclotron radius. The closed magnetic square billiard in turn, corresponding to $a=1$, is integrable in the low- and high-field limits but shows a mixed dynamics for intermediate values of $r_{\mathrm{c}}$ [15]. The full transition from the free channel to the square billiard, for an intermediate field $\left(r_{\mathrm{c}}=2\right)$, is illustrated by figure 5 .
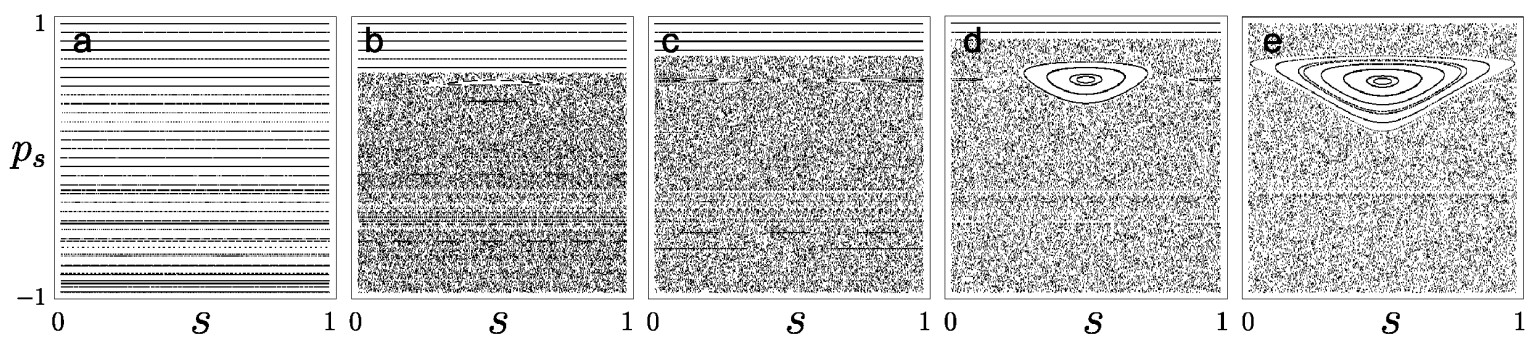

Figure 5. Phase-space portraits (sector I only, cf. figure 4) for $r_{\mathrm{c}}=2$ and $a=0$ (a), 0.25 (b), 0.5 (c), 0.75 (d), 1 (e).

A special feature related to the rectangular geometry of the billiard is the occurrence of periodic orbits in pairs, one of them stable, the other unstable, such as figures $3 \mathrm{a}$ vs. b and c vs. d. They emerge through a tangent bifurcation [34 from periodic orbits of the integrable motion at zero field that fulfill certain symmetry conditions, e.g., a diamondshaped orbit in the case of figure $3 \mathrm{a} / \mathrm{b}$. Switching on the field, each straight segment of the orbit can then be replaced by circular arcs with either curvature, convex or concave from outside, without affecting the topology of the orbit, hence their appearance in pairs [25]. Concave orbit sections tend to stabilize the orbit (panels a, c in figure 3), while convex sections tend to destabilize it (panels b, d). This mechanism is subject to strong pruning at finite values of the field and may be restricted to extremely small curvature for longer periodic orbits.

Lifting the condition $l=w$ of square unit cells affects periodic orbits with $C_{4}$ symmetry. They do not disappear immediately for $l \neq w$, but become deformed and eventually are eliminated through pruning. At the same time, towards the extremes $l \gg w$ or $l \ll w$, bouncing-ball orbits between the horizontal or vertical walls (items $\mathrm{f}, \mathrm{g}$ in figure 3), respectively, become dominant. While they themselves form a set of measure zero (straight lines in the Poincaré section 2), they tend to induce a reduction of the Lyapunov exponent on average of chaotic trajectories in the surrounding chaotic sea. For the effects on transport of lifting the square symmetry of the unit cell, see section 2.4.

For intermediate values of $r_{\mathrm{c}}$ and $a$, the classical motion in the billiard is characterized by the coexistence of regular and chaotic regions in phase space, as reflected in the Poincaré sections, figure 2, The regular components come in two forms: trajectories that result from a mere deformation of free motion along the open part of the channel, and therefore represent a continuum of momenta, cf. panel $\mathrm{m}$ in figure 3 , and regular islands centered over stable periodic orbits that can be characterized by 
rational winding numbers (panels a, c, e in figure 3). In topological terms, the latter are analogous to smoothly contractible orbits in systems with a cylindrical phase space like the kicked rotor [30], while the former ones cannot be contracted to a point. Both types of phase-space components contribute in qualitatively different ways to directed transport, see section 2.4 .

Based on these distinctions we decompose the energy shell $\mathcal{E}$ into a complete set of disjunct invariant manifolds $\mathcal{M}_{i}$ :

$$
\mathcal{E}=\cup_{i} \mathcal{M}_{i}, \quad \mathcal{M}_{i} \cap \mathcal{M}_{j}=\emptyset \text { for } \quad i \neq j
$$

We select the invariant manifolds Regular ${ }^{0}$, Regular ${ }^{+}$, Regular ${ }^{-}$(regular with zero, positive, and negative winding number, resp.), and Chaotic. This classification is to a certain degree arbitrary and could well be finer or cruder. It is, however, appropriate for the analysis of transport properties in the subsequent section.

\subsection{Directed transport}

Directed transport in a billiard with compact phase space is restricted by a sum rule discussed in [6, 7]. It implies that on average over the total phase space, all directed currents vanish. In the present case of an autonomous system, the energy shells form compact invariant subsets of phase space, so that the sum rule even applies per energy shell $\mathcal{E}$ and reads

$$
\begin{aligned}
\tau_{\mathcal{E}} & =\sum_{i} \tau_{\mathcal{M}_{i}}=0 \\
\tau_{\mathcal{M}_{i}} & =\lim _{t \rightarrow \infty} \frac{1}{t} \int_{0}^{t} \mathrm{~d} t^{\prime} \int_{\mathcal{M}_{i}} \mathrm{~d}^{2 f-1} r \rho_{t}(\mathbf{r}) v_{x},
\end{aligned}
$$

where $\tau_{\mathcal{E}}$ is the total transport over the energy shell, $\tau_{\mathcal{M}_{i}}$ the transport per invariant set $\mathcal{M}_{i}$, and $\rho(\mathbf{r})$ is the natural measure at some point $\mathbf{r}$ on the $(2 f-1)$-dimensional energy shell. It corresponds to a homogeneous distribution over the spatial unit cell and an isotropic distribution of velocities.

This does not preclude, however, that directed transport occur within individual invariant sets $\mathcal{M}_{i}$, such as in particular for the chaotic sea. In figure 6, we compare the corresponding contributions from the chaotic sea and the regular components specified above. It is obvious that chaotic transport reflects directly the imbalance between currents in the regular regions of phase space. A dominant rôle is played by the set of non-contractible trajectories passing below the side walls, cf. figure $3 \mathrm{~m}$. They form a continuous family parameterized, e.g., by the positions $\left(x_{\mathrm{c}}, y_{\mathrm{c}}\right)$ of the center of the circular arcs making up the orbit. Its vertical component, in turn, is bounded from above by $y_{\mathrm{c}}<1-a-r_{\mathrm{c}}$. This allows us to estimate analytically the contribution to transport of the invariant set formed by these orbits, which we denominate $R_{m}$, as a function of $a$, see appendix B; the results are included as full lines in figure 6b. For

comparison, figure 6a shows the fractions of the energy shell volume $f_{i}=\int_{\mathcal{M}_{i}} \mathrm{~d}^{2 f-1} r \rho_{t}(\mathbf{r})$ pertaining to each invariant set $\mathcal{M}_{i}$ as functions of $a$. We note in passing that directed 
transport, say, in the chaotic sea would allow for a finite current to develop even from an unbiased initial condition (i.e., symmetric in $p_{x}$ ).
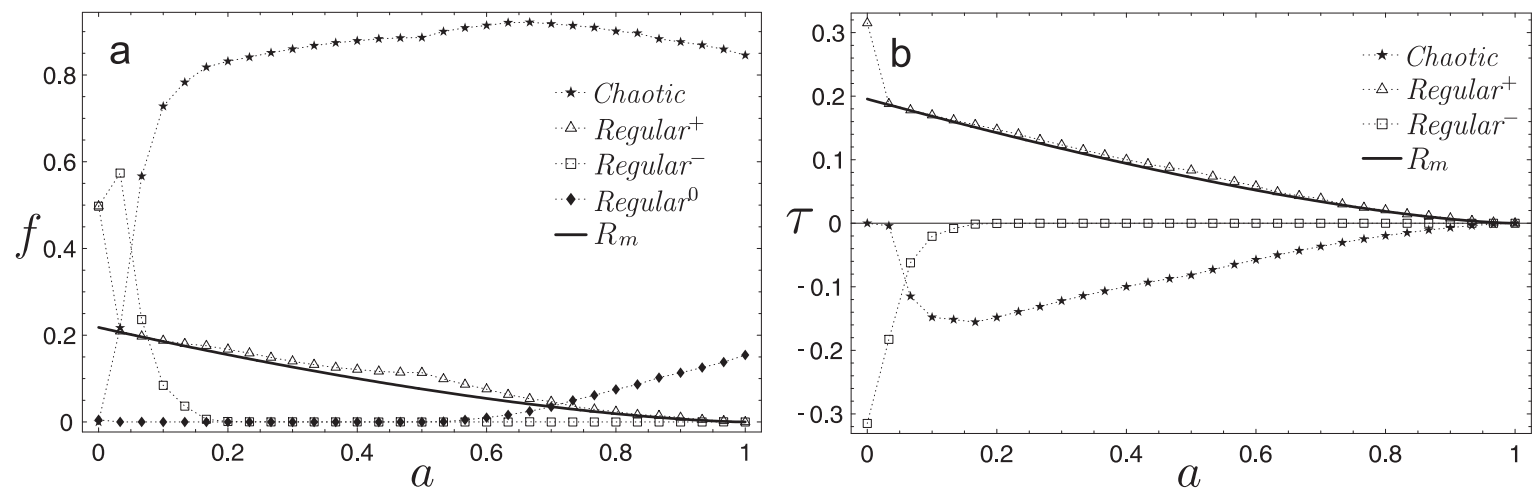

Figure 6. Fractions of the energy shell pertaining to different invariant sets $\mathcal{M}_{i}$, cf. (3) (a) and directed current $\tau$ for these invariant sets (b) vs. the barrier size $a$ at $r_{\mathrm{c}}=2$. Solid lines: analytical solutions, see appendix B.

There is no transport in the limit $r_{\mathrm{c}} \rightarrow \infty$, as it corresponds to vanishing magnetic field and thus restores time-reversal invariance. In the opposite limit $r_{\mathrm{c}} \rightarrow 0$, the entire phase space is filled with non-transporting circular orbits. Likewise, for $a=0$ transport vanishes because the billiard becomes spatially symmetric, while for $a=1$, the unit cell is closed. For $a>0$, at $r_{\mathrm{c}}=2$, the principal contribution to transport to the right comes from $R_{m} \subset$ Regular $^{+}$. The slight discrepancy between the curves corresponding to $\operatorname{Regular}^{+}$and the estimate for $R_{m}$, respectively, in figure 6 reflects the contribution by the regular island pertaining to the periodic orbit depicted in figure 3 c. The component $\operatorname{Regular}^{0}$, in turn, is dominated by the periodic orbit in $3 \mathrm{a}$; its area decreases from a maximum at $a=1$ till it vanishes at $a=0.5$ where the underlying orbit gets pruned. There is no appreciable regular transport to the left for $a>0$. The finite values of $f_{R^{-}}$we obtained for small $a$ are numerical artifacts, owed to the difficulty to distinguish regular trajectories from chaotic ones with very small positive Lyapunov exponent as they occur in this range.

The existence of the phase-space component $R_{m}$ also renders directed transport very robust with respect to deviations from the symmetry $l=w$. In either direction, $R_{m}$ is not affected as it depends only on $a$. By contrast, components like Regular ${ }^{0}$ eventually disappear with the underlying regular islands for $l \gg w$ or vice versa, but are replaced by other components with equivalent transport features. Even in the corresponding limits, the phase-space components supporting directed transport maintain a finite weight.

\section{Quantization}

Quantizing a billiard threaded by a magnetic field amounts to solving the timeindependent Schrödinger equation including a vector potential $\mathbf{A}(\mathbf{x}), \mathbf{x}=(x, y)$,

$$
\frac{1}{2 m}(-i \hbar \nabla-q \mathbf{A}(\mathbf{x}))^{2} \psi(\mathbf{x})=E \psi(\mathbf{x})
$$


with Dirichlet boundary conditions along the walls.

The vector potential $\mathbf{A}(\mathbf{x})$ is determined by the magnetic field through $\mathbf{B}=\nabla \times \mathbf{A}$ only up to a gauge. We here choose the Landau gauge [21]

$$
\mathbf{A}(\mathbf{x})=B(-y, 0)
$$

as the resulting eigenstates preserve the reflection symmetry (2). The resulting Schrödinger equation is equivalent to a Helmholtz equation with the same boundary conditions, up to a Peierls phase in its interior [35].

Among the quantities to be derived from the wave function we mention the spatial probability density

$$
\rho(\mathbf{x})=|\psi(\mathbf{x})|^{2}
$$

and the probability density current

$$
\mathbf{J}(\mathbf{x})=-\frac{\hbar}{m} \operatorname{Im}\left(\psi(\mathbf{x}) \nabla \psi^{*}(\mathbf{x})\right)-\frac{q}{m} \mathbf{A}(\mathbf{x})|\psi(\mathbf{x})|^{2}
$$

which is useful, e.g., to identify vortices, see 3.2.4, and to compare the quantummechanical probability flow with classical transport mechanisms, see 3.3 .

\subsection{Quantization algorithm}

Numerous algorithms are available for solving (6) [16, 36, 37, 38, 39, 40, 41]. We choose a finite-element technique [13] as it is optimally suited to fully exploit the simple geometry of the billiard, with Dirichlet boundary conditions along straight walls at right angles. A practical difficulty arises, however, at the free ends of the side walls that form cusps of the boundary and induce a very non-smooth behavior of the wave function in their vicinity. In order to achieve reasonable solutions even in these critical regions, we employ cubic Hermite polynomials as basis functions in the finite-element algorithm [42]. The presence of the vector potential is reflected in a finite Peierls phase corresponding to the magnetic flux through each cell of the grid underlying the finite-element algorithm [35].

Another non-standard aspect in this context is the periodicity (11) of the billiard. It is appropriately taken into account in the quantum mechanical treatment by working in the Bloch formalism [43]. Assuming a total system size of $M$ unit cells with overall periodic boundary conditions, it implies in particular a periodicity condition from cell to cell

$$
\psi_{k_{x}}(x+1, y)=\psi_{k_{x}}(x, y) \exp \left(i k_{x}\right) ;
$$

where $k_{x}$ is the Bloch number (equivalent to the quasimomentum and the Bloch phase) given by

$$
k_{x}=\frac{2 \pi m}{L}=\frac{2 \pi m}{M l}
$$

where $L=M l$ is the total billiard length and $m=0,1,2, \ldots, M-1$. This condition allows to solve the Schrödinger equation over an effective area that corresponds to the unit cell only, by requiring a fixed phase difference given by (10) between the open 
boundary sections along its left and right edges, respectively, where Dirichlet boundary conditions do not apply.

Resuming, the finite-element approach has to be complemented with (i), a local phase accumulated around the circumference of each cell of the grid, and (ii), a global phase difference between the open sections of the billiard's unit cell. Both phases are to be considered parameters of the system, corresponding to the magnetic flux and the Bloch phase, respectively.

Details of the algorithm are deferred to appendix C.

\subsection{Spectrum and eigenstates}

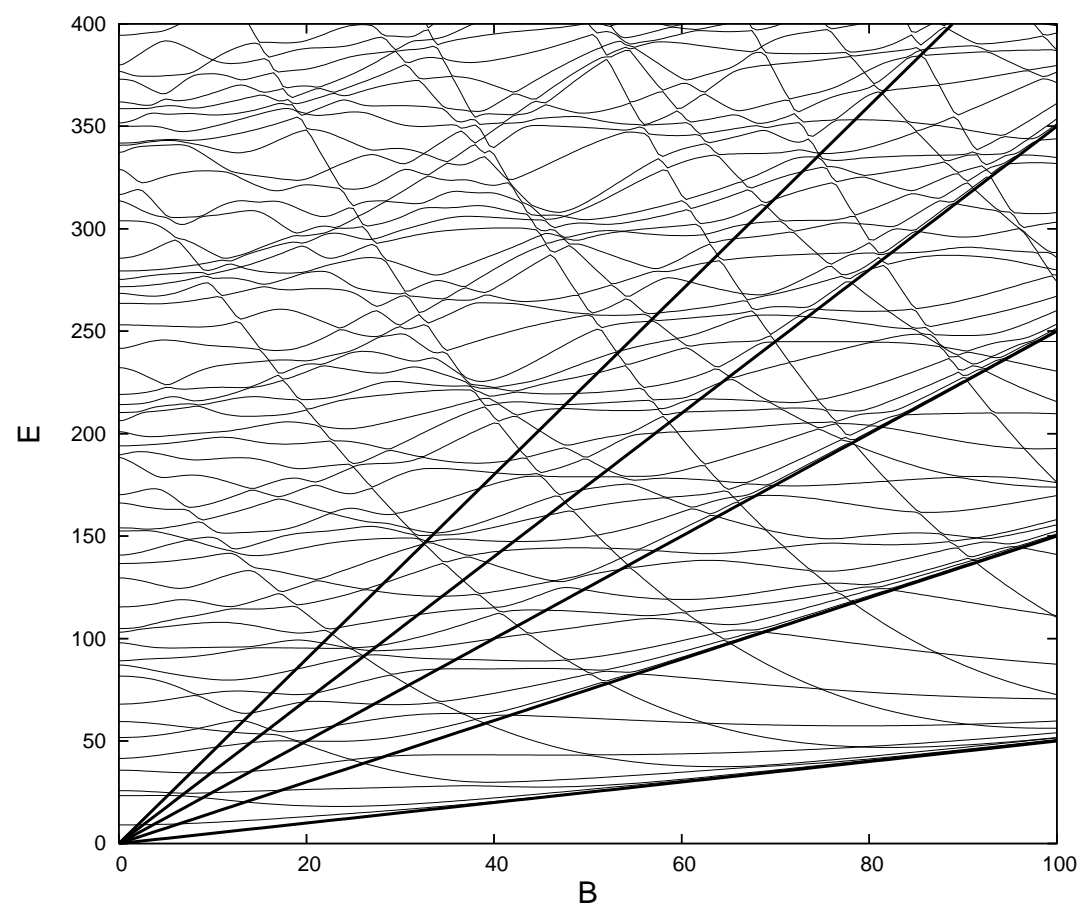

Figure 7. Eigenenergies $E$ vs. magnetic field $B$ for $a=0.5$, at $k=0$. Bold straight lines indicate Landau levels (12).

3.2.1. Landau levels For a two-dimensional electron gas, i.e., charged particles moving in a plane perpendicular to a constant homogeneous magnetic field but otherwise free, the eigenenergies are given by

$$
E_{n}=(n+1 / 2) \hbar q B,
$$

known as Landau levels, and the eigenstates are analogous to those of a two-dimensional symmetric harmonic oscillator [44]. They are characterized by two spatial scales, one given by the cyclotron radius $r_{\mathrm{c}}=p / q B$, as for the corresponding classical motion, the other by the magnetic length $b=\sqrt{2 \hbar / q B}$ [45], the radial width of eigenstates concentrated along circles of radius $r_{\mathrm{c}}$. These Landau states become the dominant feature of the quantum billiard in the limit of strong magnetic field, i.e., for $B \gg p / q$ 
and $B \gg \hbar / q$. This expectation is confirmed by the data shown in figure 7 . While for low field, we observe the spectral structures like level repulsion typical for a classically chaotic system, the eigenenergies tend to accumulate towards the Landau levels (bold lines) in the high-field limit. Their influence remains visible in the band structure as marked concentrations of bands, in particular for strong field, as visible in figure $8 \mathrm{c}$ below.

3.2.2. Band spectrum The fingerprints of directed transport in the band spectrum are amply discussed in [6, 7]. A crucial concept for their understanding is that of diabatic bands, coherent large-scale structures in the spectrum that are formed by connecting sections of actual ("adiabatic") bands across small gaps ("avoided crossings"). They can be directly associated to the current-carrying manifolds in classical phase space. Regular islands with non-zero winding numbers correspond to approximately straight lines in the spectrum which, upon connecting them according to the periodicity of the Brillouin zone, exhibit the same winding number as the underlying classical island. Diffusion with superposed drift in the chaotic sea is reflected in wiggly diabatic bands with an average slope related to the mean transport velocity in this part of the classical phase space. Moreover, among the "chaotic bands" we observe the phenomenon of level repulsion characteristic for classically chaotic quantum systems [46].
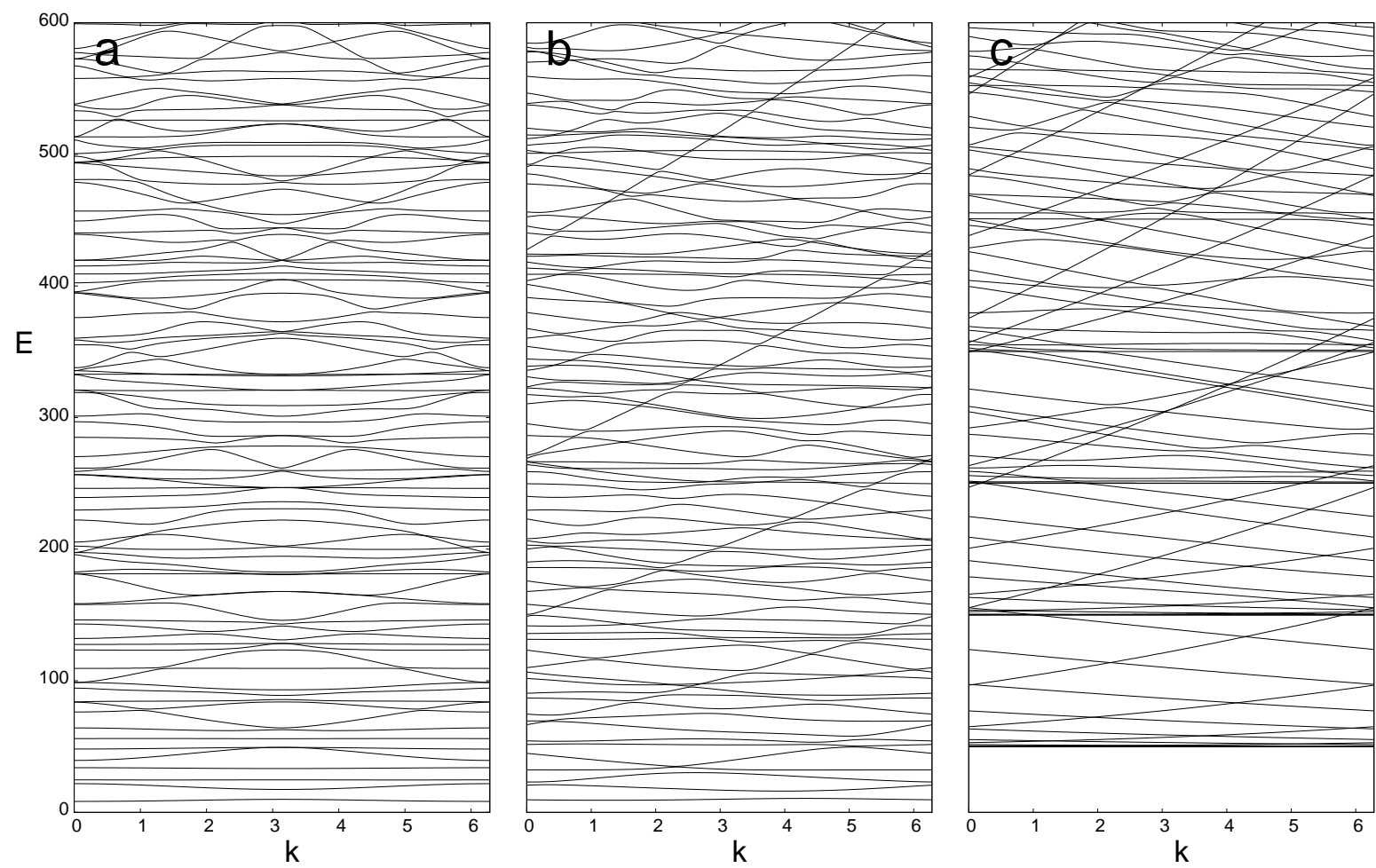

Figure 8. Lowest 90 energy bands for $a=0.5$ and $B=0$ (a), $B=10$ (b), and $B=100(\mathrm{c})$.

As mentioned in 2.4, a dominating feature of directed transport in the magnetic billiard is the contribution by non-contractible open trajectories that correspond to 
quasi-free motion through the gaps left between the side walls and the lower wall of the channel. As discussed above, the winding numbers of these trajectories form a continuum and depend on energy, by contrast to the regular islands whose rational winding numbers reflect their topology and therefore may be called structurally stable. Indeed, we observe structures in the band spectrum, cf. figures 8b-c, that resemble the parabolic bands pertaining to quasi-free particles moving in a crystalline potential 43. The conspicuous, apparently straight lines with positive slope appearing in figures 9]b-c are closer to features seen in quantum ratchets [6, 7] but in fact also show a slight curvature. They belong to the same category as those in figures $8 \mathrm{~b}-\mathrm{c}$ and can be associated to the classical phase space component $R_{m}$. At the same time, we do observe some approximately straight diabatic bands with negative slope that pertain to very small regular islands with winding number $<0$. By contrast, a bias of the mean slope of the remaining wiggly bands is not so easy to discern with the unaided eye. These features have in common that they are incompatible with the familiar reflection symmetry of energy bands with respect to $k=\pi$ (as in figure 8 a) - this is indeed how the breaking of TRI by the magnetic field is reflected in the band structure. They are of particular relevance for directed transport, as discussed in section 3.3 below.
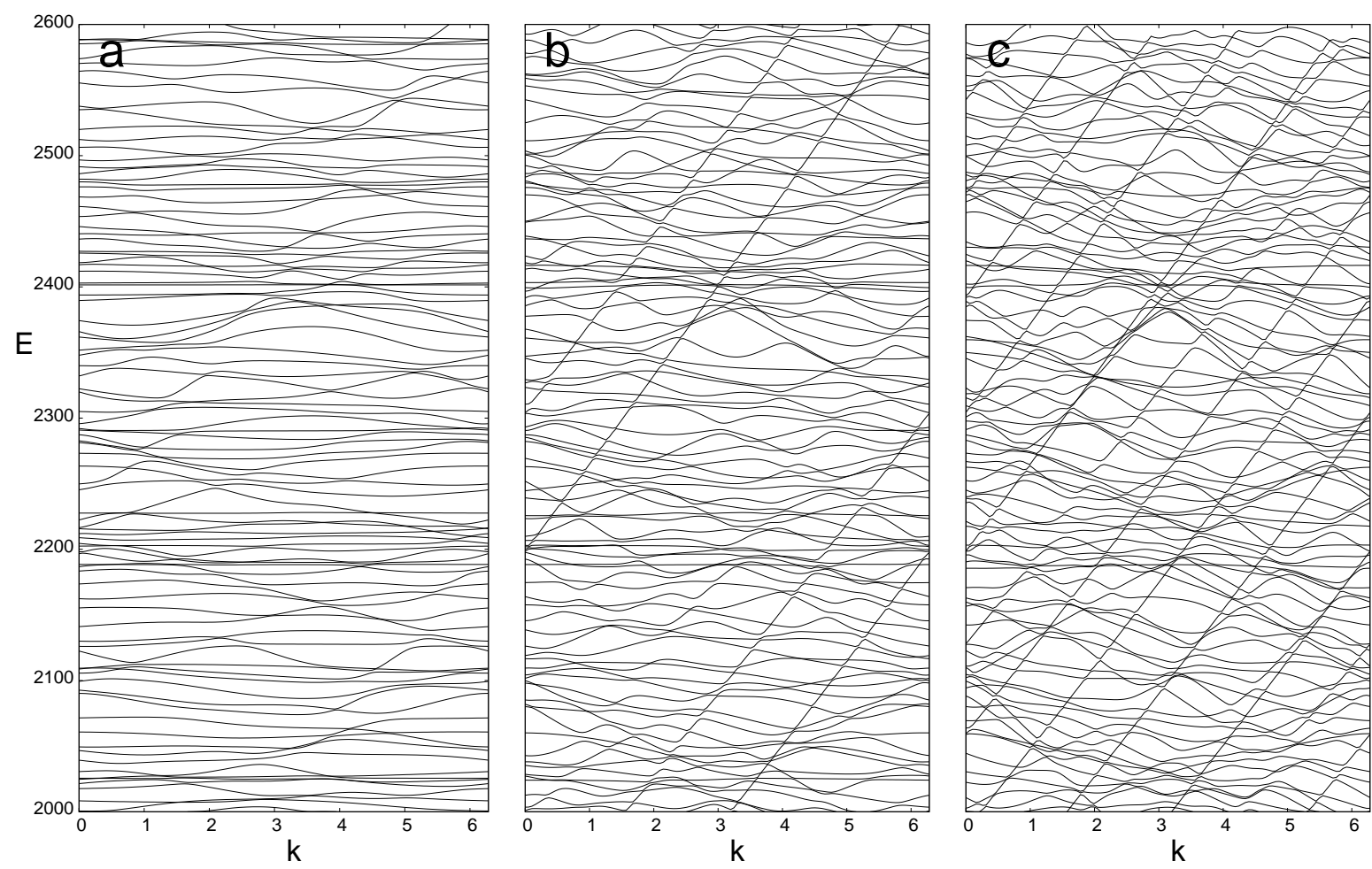

Figure 9. Energy bands $E_{n}$ with $300 \leq n \leq 400$ for $B=10$ and $a=0.8$ (a), 0.5 (b), and $0.2(\mathrm{c})$.

3.2.3. Bloch states We analyze the eigenstates of the periodic billiard, that is, its Bloch states, to resolve the flow structures underlying global transport properties, to 
identify fingerprints of the corresponding classical dynamics, and to observe vortices of the quantum flow as special quantum features associated with directed transport in the billiard [47. In figures 10 and 11, we simultaneously show the probability density and flow as well as node lines of the real and imaginary parts of the wave function and its phase-space representation in terms of Husimi functions.
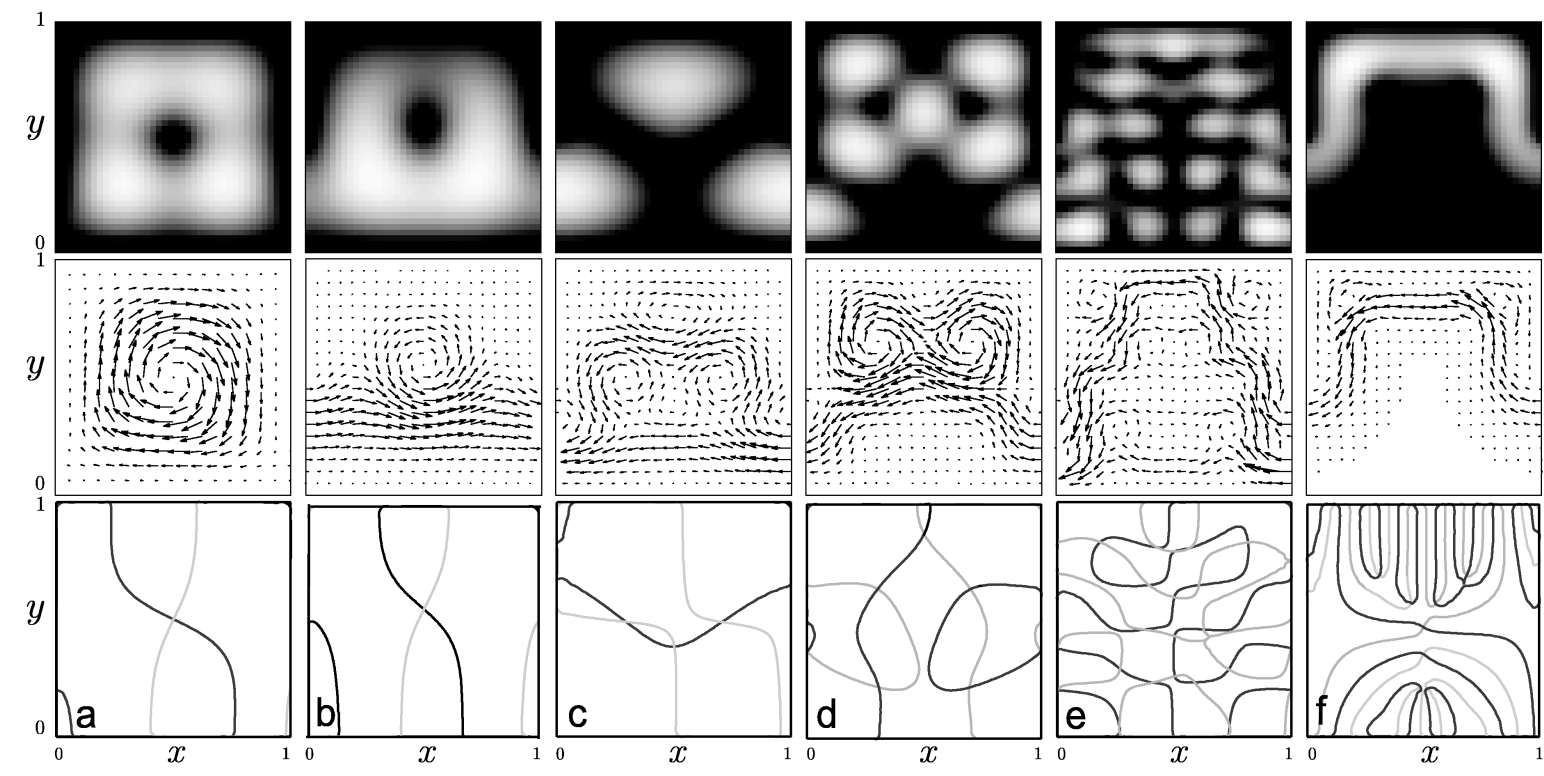

Figure 10. Examples of eigenstates represented by their probability density (8) (upper row, greyscale from black $\equiv$ zero to white $\equiv$ maximum) and flow, (9) (middle row), and nodelines (lower row) of the real (black) and imaginary parts (grey) of the wavefunction. Parameters are, $B=10$, (a-e), 100 (f), $a=0.8$ (a), 0.5 (b-f), $k=0$ (a-c), $\pi$ (d-f), and energy level index, from a to f, $n=3,2,3,8,24,11$.

The Husimi functions presented in the third row of figure 11 have been constructed following the approach detailed in [10, 11]. It reduces the system's four-dimensional phase space, exploiting the approximate factorization of the wave function near the lower horizontal wall (sector I in figure 11) into $x$ - and $y$-dependences, to a Poincaré surface of section spanned by the two dimensions $x$ and $p_{x}$.

The density distributions already allow us to classify the states according to a number of pertinent categories. For parameter values close to the corresponding limits, we observe states that resemble the solutions of the Schrödinger equation for a closed square billiard (10a) or a free channel (11b), respectively. In particular, so-called bouncing-ball states $(11 \mathrm{~d})$ pertain to these categories. Even for relatively large openings between the cells, we find states with a marked minimum across the gap (10) which therefore belong to the first category mentioned above. By contrast, the continuity equation for the probability flow implies that states supporting strong directed transport tend to exhibit high probability density and strong flow through the openings (10k, d, 11a,b). Where the flow remains well bundled even within the cell, it tends either to wind along the upper wall and sidewalls (10f), if transport is to the left, or to adhere 


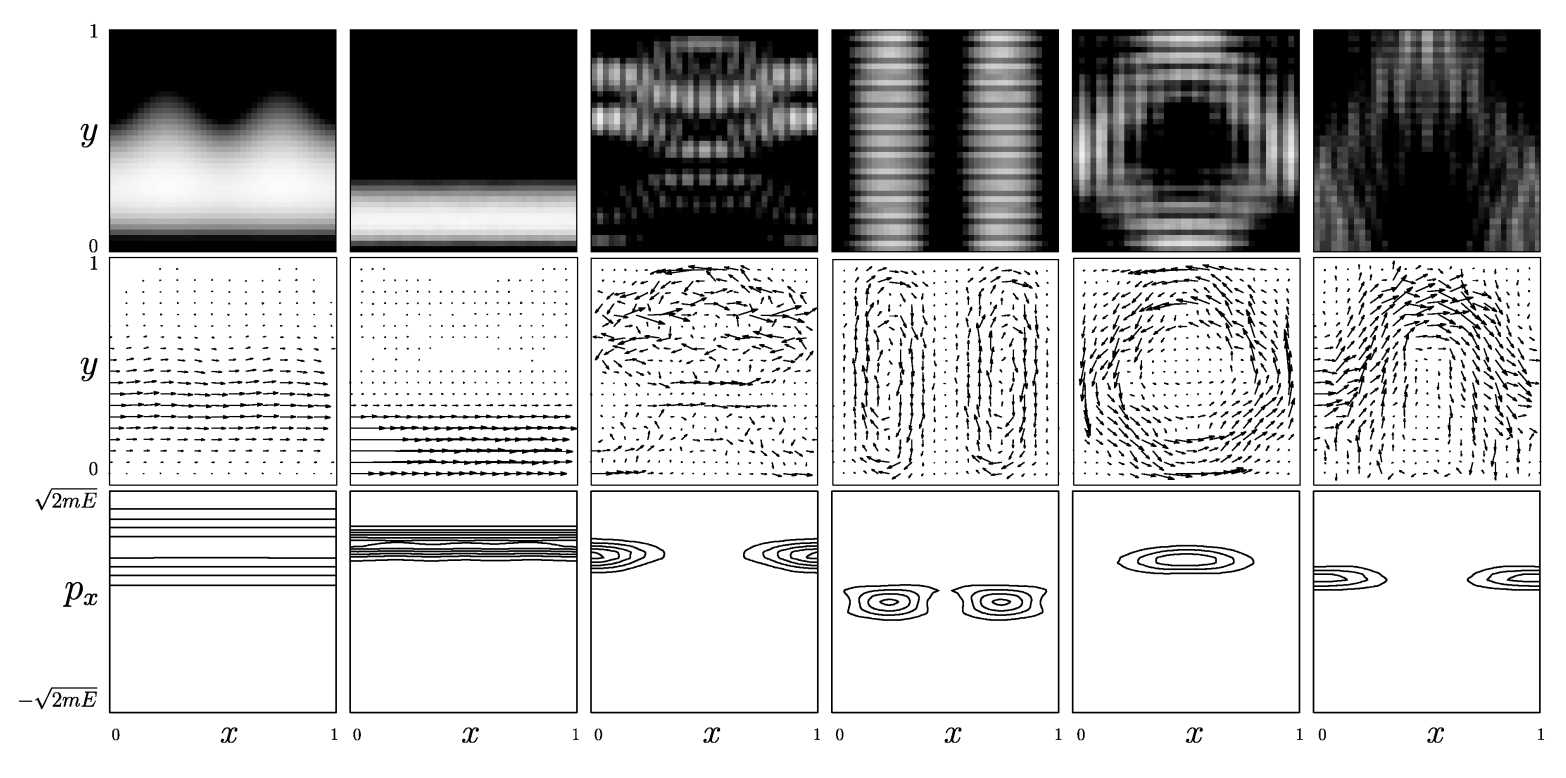

Figure 11. Examples of eigenstates represented by their probability density (8) (upper row, greyscale from black $\equiv$ zero to white $\equiv$ maximum) and flow, (9) (middle row) and Husimi functions in the plane $\left(x, p_{x}\right)$ along the lower wall of the billiard (lower row), see text for details. Parameters are $B=10, k=0$ and, from a to f, $a=0.25,0.5,0.5$, $0.5,0.8,0.2$, and energy level index $n=2,128,167,169,201,322$, corresponding to eigenenergies $E=20.25,860.6,1126,1134,1349,2101$.

to the lower wall (11b), in the opposite case. We can characterize these states as edge states [21, 31, 33], borrowing a concept from the context of the quantum Hall effect. In classical terms, in turn, they can be associated to so-called skipping orbits that creep along the walls, as they occur in the high-field regime, cf. figure 3 .

Energy eigenstates with probability densities and flows concentrated near the lower wall $(11 \mathrm{1}, \mathrm{b})$ are related to the phase-space component $R_{m}$. Indeed, their current patterns resemble the corresponding trajectories (figure $3 \mathrm{~m}$ ) and their Husimi functions tend to localize over the corresponding invariant tori. Other fingerprints of the classical phasespace flow can be observed in the Husimi functions in figures 11k, d,e,f as they concentrate over the orbits in figures 3 c,g, a,i, respectively.

3.2.4. Vortices, dia- and paramagnetism By analogy to a hydrodynamic flow with the same boundary conditions, one expects vortices to occur in the quantum-mechanical probability flow in the range $0<a<1$, in particular for $a \lesssim 1$. Experimental results for microwave billiards as systems analogous to quantum billiards [47, 48] and theoretical results for mesoscopic systems [49] support this expectation. Writing the quantum probability current (9) in the form

$$
\mathbf{J}(\mathbf{x})=\rho(\mathbf{x})(\hbar \nabla S(\mathbf{x})-q \mathbf{A}(\mathbf{x}))
$$

where $S(\mathbf{x})$ denotes the phase of the wavefunction, we identify the second factor on the rhs as a velocity

$$
\mathbf{v}(\mathbf{x})=\hbar \nabla S(\mathbf{x})-q \mathbf{A}(\mathbf{x})
$$


This expression shows that the magnetic field contributes to the formation of vortices directly and independently of the quantum phase. The difference between the two contributions is brought out even more clearly if we consider the corresponding vorticity (synonymous to the fluxoide in the context of superconductivity), defined as the line integral of $\mathbf{v}(\mathbf{x})$ around a closed contour $\Gamma$,

$$
\oint_{\Gamma} \mathrm{d} \mathbf{x} \cdot \mathbf{v}(\mathbf{x})=\hbar \oint_{\Gamma} \mathrm{d} \mathbf{x} \cdot \nabla S(\mathbf{x})-q \oint_{\Gamma} \mathrm{d} \mathbf{x} \cdot \mathbf{A}(\mathbf{x})=q\left(\Phi_{l}-\Phi_{\mathrm{mag}}\right) .
$$

Here, $\Phi_{\operatorname{mag}}$ is the magnetic flux of the external field enclosed in the contour. By contrast, the term $\Phi_{l}$ indicates topological defects of the phase of strength $2 \pi l, l$ integer. This discretization is equivalent to the quantization of the magnetic flux $\Phi_{l}=2 l \pi \hbar / q$ generated by the vortex. This induces a different behavior of the vorticity for the two types of vortices upon contracting the contour towards the center of the vortex: In the absence of singularities of the external field, $\Phi_{\text {mag }}$ then reduces to zero and so does the vorticity, while at topological defects the vorticity remains finite in the limit and therefore requires $\mathbf{v}$ to diverge. In order that the flow $\mathbf{J}=\rho \mathbf{v}$ remain finite in turn, this implies that the density $\rho(\mathbf{x})$ decay with decreasing distance $r$ from the vortex at least as $r$, that is, possess a first-order zero at the center, providing another criterion to distinguish the two classes.

Moreover, topological defects can occur with either sign, anti-clockwise or clockwise, corresponding to a paramagnetic or diamagnetic response to the external field, respectively. The sign of $\Phi_{\mathrm{mag}}$ depends on the orientation of the magnetic field; it always gives rise to diamagnetic response, that is, in our case, to clockwise vortices.

In figure 12, we show three eigenstates featuring the three classes of vortices mentioned above, a diamagnetic (panel a) and a paramagnetic (b) topological defect, and a non-quantized diamagnetic vortex (panel c). We observe that indeed, the topological defects are associated with isolated nodes of the density, while the non-quantized vortex typically sits close to a maximum of $\rho(\mathbf{x})$. Superposing graphs of the flow $\mathbf{J}(\mathbf{x})$ with nodelines of $\operatorname{Re} \psi(\mathbf{x})$ (black bold lines) and $\operatorname{Im} \psi(\mathbf{x})$ (grey) demonstrates that nodeline crossings, i.e., phase defects, coincide with vortices. Except for their intersections, these nodelines are gauge dependent and therefore have no physical significance. Their orientation and density, however, reflects the probability flow and serves as an alternative way to visualize it. For an energy eigenstate featuring both types of vortices, see figure 10k. In our case, and by contrast, e.g., to microwave billiards [48], there is no reason for vortices to occur pairwise (one dia-, one paramagnetic) or to be accompanied by a pair of saddles (hyperbolic points) of the flow. Our system therefore corresponds to a different universality class as concerns vortex statistics. We shall however not pursue this subject any further.

\subsection{Directed quantum transport}

On general grounds based on the correspondence principle, we expect to find evidence of directed transport in the quantized billiard, of comparable magnitude as in its 

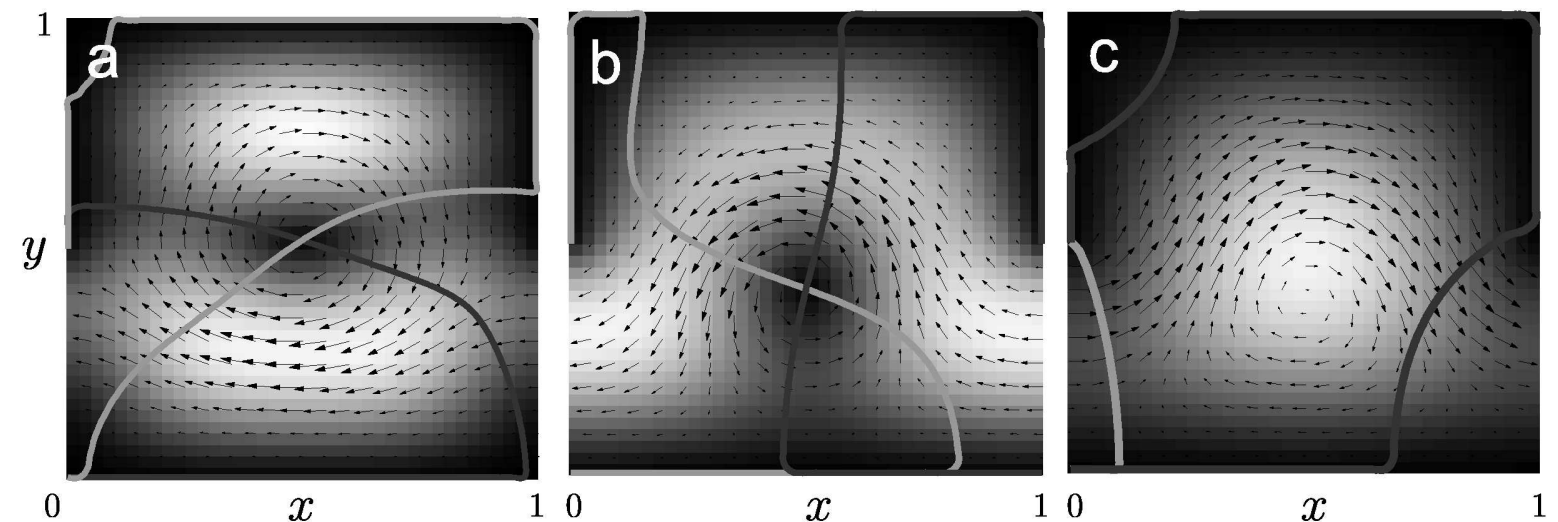

Figure 12. Examples of eigenstates featuring a diamagnetic (a) and a paramagnetic (b) topological defect, and a diamagnetic non-quantized vortex (c), represented by their probability density (8) (greyscale from black $\equiv$ zero to white $\equiv$ maximum) and flow (9), superposed with nodelines of the real (black) and imaginary parts (grey) of the wavefunction. Note the coincidence of the quantized vortices (panels a,b with quantum numbers $l=-1,1$, resp.) with zeros of the density and nodeline crossings (phase defects). Parameters are $B=10, a=0.5, k=\pi$ and, from a to c, energy level index $n=3,2,1$.

classical counterpart, at least in the semiclassical parameter regime. This expectation has been amply confirmed in the case of ratchets [6, 7]. Moreover, the examples of probability densities and probability flows in figures 10 and 11 clearly show that features of the classical dynamics relevant for transport, e.g., periodic orbits, are reflected in the quantum system. In particular, the phase-space component $R_{m}$ decisive for the occurrence of classical directed currents also dominates quantum transport and renders it robust, e.g., against deviations from square unit cells $l=w$.

Adopting an approach developed in [6, 7], we here present less direct though more comprehensive evidence for directed transport. According to Bloch theory, the mean velocity of a wavepacket prepared in the energy band $E_{n}(k)$ at quasimomentum $k$ is given by the band slope at this point [43],

$$
\bar{v}_{n}(k)=\frac{1}{\hbar} \frac{\mathrm{d} E_{n}(k)}{\mathrm{d} k} .
$$

Directed transport can therefore be assessed on basis of the band structure, in particular of the statistics of band slopes (level velocities). In figure 13 we present a histogram of band slopes obtained for the same parameter values and the same energy window as underlies figure 9k. The two most prominent features of the band structure are clearly reflected in the histogram, approximately straight diabatic bands with a positive slope $\approx 66$, corresponding to the phase-space component $R_{m}$ of the classical system, and wiggly bands with negative slope on average, corresponding to chaotic drift towards the left: They appear as a relatively marked peak at the expected value $\bar{v} \approx 66$, in very good agreement with the mean velocity $\bar{v}_{m}=66.16$ of the component $R_{m}$ in the relevant energy range $2000 \leq E \leq 2600$, and a broad approximately Gaussian background biassed towards $\bar{v}<0$, respectively. The global mean velocity vanishes 


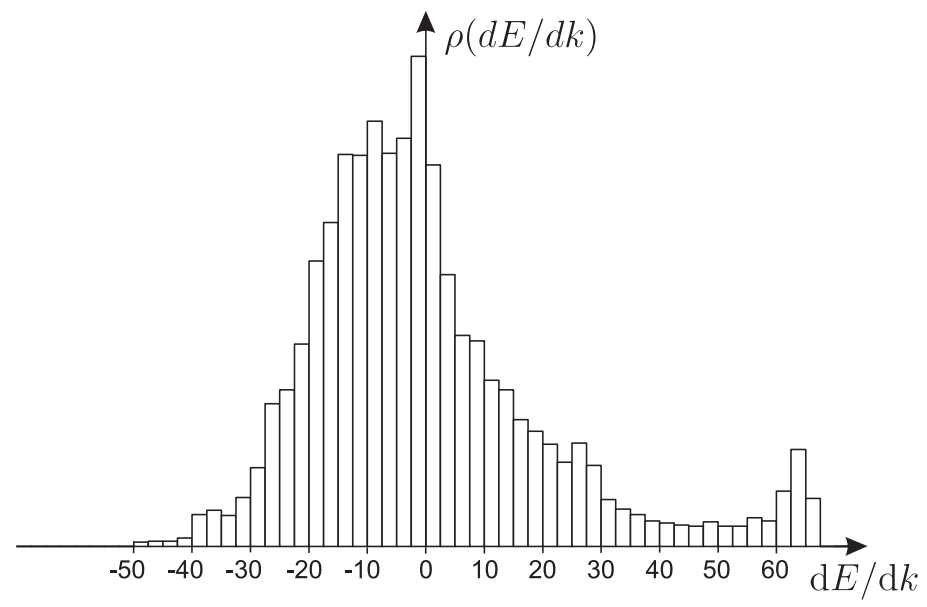

Figure 13. Histogram of the band slope (16) for energy bands $E_{n}$ with $300 \leq n \leq 400$ at $B=10$ and $a=0.2$, as shown in figure 9 c.

within numerical accuracy, reflecting the quantum analogue of the classical sum rule (4) for directed transport [6, 7]. The conspicuous peak at $\bar{v}=0$ may be associated to the contribution of the avoided crossings. All in all, our histogram closely resembles the analogous figure 7 in [7] (except for a sign reversal of the velocities).

\section{Conclusion}

Billiards emerged as mathematically-minded models for classical and quantum complex dynamics. Yet they inspired a surprisingly versatile range of laboratory experiments, ranging from lateral semiconductor superlattices [20] to microwave billiards [50, 51]. It is therefore not surprising that a more recent development in classical complex dynamics, directed transport in Hamiltonian ratchets, is also making its way from one-dimensional but time-dependent models [6, 7] to billiards, with their specific mathematical beauty and diverse experimental applications.

In this case, two of the functions fulfilled in ratchets by the external driving are taken over by a static magnetic field, namely rendering the dynamics partially chaotic and breaking time-reversal invariance. This allows to keep the geometry of the billiard exceedingly simple, a setup readily sketched on squared paper. The elementary shape also greatly facilitates the quantization of the system, using finite-element methods that appear to be taylor-made for this application.

The mechanisms responsible for directed transport in the classical billiard proposed in this paper largely carry over to the quantum system and are manifest, for example, in its band structure. A consequence of quantum transport not immediately anticipated from the classical dynamics is, e.g., the occurrence of vortices of the probability flow that coincide with phase defects of the wavefunction and correspond to a dia- or paramagnetic response of the system, respectively.

This work leaves ample space for complementary research. By adding an ac driving, 
we can generate a hybrid between an autonomous magnetic billiard and a ratchet. Even an adiabatic modulation of the magnetic field strength, without invoking any electric field, would open further possibilities to attain directed transport.

The restriction of the infinite billiard chain to a section of finite length with leads attached to both sides yields a scattering system, a configuration closer to experimental realizable setups, for example a ballistic two-dimensional electron gas in a semiconductor superlattice imposed by gate voltages or by lithography. As a closely related aspect, this setup would provide an additional way to break TRI, by incoherent decay processes occurring in the electron reservoirs coupled to the system, and therefore work even in the absence of the magnetic field.

In such a setup, it would even make sense to study aspects of transport under an external dc voltage bias, indicating an alternative way towards the experimental observation of fingerprints of directed transport as it occurs without driving: The response to a dc voltage would make contact with magnetotransport and magnetoresistance.

\section{Acknowledgments}

We enjoyed fruitful discussions with N Berglund, B Dietz-Pilatus, F Leyvraz, A Richter, H Schanz, T Seligman, and C Viviescas. Financial support by Volkswagen Foundation (grant I/78235) and Colciencias (grant 1101-05-17608) is gratefully acknowledged. One of us (TD) thanks for the warm hospitality and inspiring atmosphere enjoyed at Centro Internacional de Ciencias AC in Cuernavaca (Mexico) and at Institut für Kernphysik, Technische Universität Darmstadt (Germany).

\section{Appendix A: Classical Poincaré map}

To simulate the dynamics of the billard, we employ a geometric algorithm based on the coordinates $(\mathbf{x}, \alpha)$, constructed so as to minimize rounding errors. In order to obtain the Poincaré map, we need in addition the two transformations that lead from $(\mathbf{x}, \alpha)$ to the Birkhoff coordinates $(s, \cos \alpha)$ and v.v. The algorithm consists of the following principal steps:

- For a given initial condition $\left(\mathbf{x}_{0}, \alpha_{0}\right)$ and a fixed value of the cyclotron radius $r_{\mathrm{c}}$, calculate the coordinates $\mathbf{x}_{\mathrm{c}}$ of the circle on which the particle is moving.

- Find the set $\left\{\mathbf{x}_{l}\right\}$ of intersections of this circle with the circumference of the unit cell and its periodic continuation, i.e., with horizontal walls (lines $y=0$ and $y=1$ ), vertical walls $(x=n, n$ integer, with $1-a \leq y<1)$ and the open unit-cell boundary $(x=n$ with $0 \leq y<1-a)$.

- Identify among the $\mathbf{x}_{l}$ the value, call it $\mathbf{x}_{l_{0}}$, that corresponds to the initial condition $\mathbf{x}_{0}$ itself, taking into account that due to rounding errors, $\mathbf{x}_{l_{0}}$ and $\mathbf{x}_{0}$ might be not exactly identical. 
- Calculate the angles $\beta_{l}$ between the vector $\overrightarrow{\mathbf{x}_{\mathrm{c}} \mathbf{x}_{0}}$ and each one of the vectors $\overrightarrow{\mathbf{x}_{\mathrm{c}} \mathbf{x}_{l}}$ with $l \neq l_{0}$, and rearrange the intersections in the order of increasing $\beta_{l}$, from $\beta_{l_{0}}$ onwards.

- Select the first intersection in forward direction along the trajectory, i.e., the one pertaining to $\beta_{1}$ (the smallest after reordering), as the actual next inflection point of the trajectory. If it corresponds to a reflection at a wall, the angle at exit is $\alpha_{1}=2 \psi_{1}+\beta_{1}-\alpha_{0}$ where $\psi_{1}$ defines the orientation of the wall hit by the trajectory. If it corresponds to passing to the left or right adjacent cell, the angle at exit is $\alpha_{1}=\alpha_{0}-\beta_{1}$.

Note that with growing cyclotron radius, the number of intersections to be taken into account in the second step increases, rendering the algorithm slower.

\section{Appendix B: Component $R_{m}$}

The phase-space component $R_{m}$ is formed by skipping orbits that only hit the lower wall, cf. figure $3 \mathrm{~m}$. For a given value of $r_{\mathrm{c}}$, this family is bounded by those trajectories that touch the lower tip of the vertical walls at their climax. Taking this condition into account, one readily shows that $R_{m}$ is delimited within the energy shell by

$$
R_{m}=\left\{\mathbf{r}\left|y<a^{\prime}, \quad\right| \alpha \mid<\arccos \left(1-\left(a^{\prime}-y\right) / r_{\mathrm{c}}\right)\right\},
$$

where $\mathbf{r}=\{x, y, \alpha\}$ is a point on the energy shell and $a^{\prime}=1-a$. Starting from (A.1), we can estimate analytically the fraction of the energy shell occupied by $R_{m}$ as

$$
f_{R_{m}}=\left(a^{\prime}-r_{\mathrm{c}}\right)\left(\frac{1}{2}+\frac{1}{\pi} \arcsin \left(\frac{a^{\prime}-r_{\mathrm{c}}}{r_{\mathrm{c}}}\right)\right)+\frac{1}{\pi} \sqrt{a^{\prime}\left(2 r_{\mathrm{c}}-a^{\prime}\right)} .
$$

Evaluating (5) for the component $R_{m}$, we obtain for its contribution to directed transport

$$
\tau_{R_{m}}=\frac{\sqrt{2 E}}{2 \pi} \sqrt{\frac{2 a^{\prime}}{r_{\mathrm{c}}}-\frac{a^{\prime 2}}{r_{\mathrm{c}}^{2}}}\left(a^{\prime}-r_{\mathrm{c}}-\frac{2 r_{\mathrm{c}}^{2}}{\sqrt{a^{\prime}\left(2 r_{\mathrm{c}}-a^{\prime}\right)}} \arctan \left(-\sqrt{\frac{a^{\prime}}{2 r_{\mathrm{c}}-a^{\prime}}}\right)\right) .
$$

\section{Appendix C: Finite-element quantization algorithm}

In order to solve the time-independent Schrödinger equation for the billiard, we cover the unit cell with a homogeneous square grid of $N \times N$ nodes and express the wavefunction in terms of bicubic Hermite polynomials [42], centered at each node. Substituting this expression in (66) and applying Galerkin's criterion [42] leads to a generalized eigenvalue problem where the Hamiltonian and overlap matrices have dimensions of the order of $4 N^{2}$. We solve it numerically using the LAPACK routine ZHEGVX. The periodic boundary conditions with Bloch phase are taken into account as additional phase factors at those nodes that coincide with the open sections II and VI of the cell boundary, see figure 1 .

This method is subject to limitations both in energy and in magnetic field strength. The former arise by the small-scale oscillations of high-lying eigenstates which eventually 
are no longer resolved by the mesh. The latter comes about by the typical extension of Landau states, inversely proportional to $B$, which also leads to a trade-off with the mesh size.

\section{References}

[1] Maddox J 1993 Nature 365203.

[2] Astumian R D and Bier M 1994 Phys. Rev. Lett. 721766.

[3] Jülicher F, Ajdari A, and Prost J 1997 Rev. Mod. Phys. 691269.

[4] Reimann P 2002 Phys. Rep. 36157.

[5] Jung P, Kissner J G, and Hänggi P 1996 Phys. Rev. Lett. 763436.

[6] Schanz H, Otto M-F, Ketzmerick R, and Dittrich T 2001 Phys. Rev. Lett. 87070601.

[7] Schanz H, Dittrich T, and Ketzmerick R 2005 Phys. Rev. E 71026228.

[8] Flach S, Yevtushenko O, and Zolotaryuk Y 2001 Phys. Rev. Lett. 842358.

[9] Smilansky U 1990 private communication.

[10] Crespi B, Perez G, and Chang S-J 1993 Phys. Rev. E 47986.

[11] Luna-Acosta G A, Na K, Reichl L E, and Krokhin A A 1996 Phys. Rev. E 533271.

[12] Robnik M and Berry M V 1985 J. Phys. A: Math. Gen. 181361.

[13] Štiková H, Smrčka L and Isihara A 1989 J. Phys.: Cond. Mat. 17965.

[14] Meplan O, Brut F, and Cignoux C 1993 J. Phys. A: Math. Gen. 26237.

[15] Berglund N and Kunz H 1996 J. Stat. Phys. 8381.

[16] Nakamura K and Thomas H 1988 Phys. Rev. Lett. 61247.

[17] Silberbauer H and Rössler U 1994 Phys. Rev. B 5011911.

[18] Fleischmann R, Geisel T, and Ketzmerick R 1992 Phys. Rev. Lett. 681367.

[19] Geisel T, Ketzmerick R, and Schedletzky O 1992 Phys. Rev. Lett. 691680.

[20] Weiss D et al 1991 Phys. Rev. Lett. 662790.

[21] Hornberger K and Smilansky U 2002 Phys. Rep. 367249.

[22] Schanz H and Prusty M 2005 J. Phys. A: Math. Gen. 3810085.

[23] Höhberger E M, Lorke A, Wegscheider W, and Bichler M 2001 Appl. Phys. Lett. 782905.

[24] Acevedo J W and Dittrich T 2003 Prog. Theor. Phys. Suppl. 150313.

[25] Acevedo J W 2004 BSc thesis (Bogota: Universidad Nacional de Colombia).

[26] Hamermesh M 1976 Group Theory (Reading, MS: Addison-Wesley).

[27] Richens P J and Berry M V 1981 Physica D 2495.

[28] Hannay J H and McCraw R J 1990 J. Phys. A: Math. Gen. 23887.

[29] Wiersig J 2000 Phys. Rev. E 62, R21.

[30] Lichtenberg A L and Lieberman M A 1992 Regular and Stochastic Motion 2nd ed, Applied Mathematical Sciences vol 38 (New York: Springer).

[31] Halperin B I 1982 Phys. Rev. B 25, 2185.

[32] Beenakker C W J and Van Houten H 1988 Phys. Rev. Lett. 60, 2406.

[33] Kramer B Quantization of Transport in Quantum Transport and Dissipation Dittrich T et al (eds) 1998 (Weinheim: Wiley-VCH) p 86.

[34] Ott E (1993) Chaos in dynamical systems 1993 (Cambridge: Cambridge University Press) p 46.

[35] Kramer B and Mašek J 1988 J. Phys. C: Solid State Phys. 21 L1147.

[36] Weisz J F and Berggren K-F 1990 Phys. Rev. B 411687.

[37] Klama S J. Phys.: Cond. Mat. 199355609.

[38] Wang Y, Wang J, and Guo H 1994 Phys. Rev. B 491928.

[39] Ji L and Berggren K-F 1995 Phys. Rev. B 521745.

[40] Blaschke J and Brack M 1997 Phys. Rev. A 56182.

[41] Hornberger K and Smilansky U 2000 J. Phys. A: Math. Gen. 332829.

[42] Prenter P M 1975 Splines and variational methods (New York: Wiley). 
[43] Ashcroft N W and Mermin N D 1976 Solid State Physics (Philadelphia: Holt-Saunders).

[44] Cohen-Tannoudji C, Diu B, and Laloë F 1977 Quantum Mechanics (New York: Wiley) p 752.

[45] Ballentine L E 1998 Quantum mechanics: a modern development (Singapore: World Scientific) ch 11.

[46] Haake F 2000 Quantum Signatures of Chaos 2nd ed (Berlin: Springer).

[47] Šeba P, Kuhl U, Barth M, and Stöckmann H-J 1999 J. Phys. A: Math. Gen. 328225.

[48] Kuhl U, Höhmann R, Stöckmann H-J, and Gnutzmann S 2007 Phys. Rev. E 75036204.

[49] Exner P, Šeba P, Sadreev A, Středa P and Feher P 1998 Phys. Rev. Lett. 801710.

[50] Stöckmann H-J and Stein J 1990 Phys. Rev. Lett. 642215.

[51] Gräf H-D et al 1992 Phys. Rev. Lett. 691296. 